\title{
Analysis of cyst fluid obtained by endoscopic ultra- sound-guided fine-needle aspiration supporting the diagnosis of a pancreatic neuroendocrine neoplasm
}

A 28-year-old man presented at the emergency ward for bronchopneumonia with hemoptysis. A computed tomography (CT) scan disclosed an incidental 18-mmwide lesion in the pancreatic tail that appeared cystic with magnetic resonance imaging, with a thick wall and a solid projection, both contrast-enhanced. Serological tumor markers were in the normal range.

Endoscopic ultrasound (EUS) evaluation showed an oval, protruding mass with a mixed solid and cystic echo structure (๑ Fig. 1).

Fine-needle aspiration (FNA) produced cystic fluid; two slides were smeared and one was stained with hematoxylin and eosin for rapid on-site evaluation. Part of the fluid was sent to the laboratory for tumor marker analysis, while the remainder was preserved in 95\% ethanol for cell block preparation. The observation by the onsite cytopathologist of a small group of cells suspected of being a pancreatic endocrine neoplasm (PEN) ( $\bullet$ Fig. 2) prompted the request for analysis of chromogranin $\mathrm{A}$ in the cystic fluid.

Cell-block sections showed discohesive epithelial cells with a plasmocytoid appearance, regular nuclear membrane, and finely granular chromatin; immunocytochemistry (ICC) results (positivity for chromogranin A and synaptophysin) confirmed the endocrine differentiation. The proliferation index with Ki-67 was positive in $<1 \%$ of neoplastic cells ( $\mathbf{F i g .} 3$ ).

The final cytological diagnosis of a neuroendocrine tumor was supported by the cyst fluid analysis, showing high levels of chromogranin A (138 ng/mL, normal range $20-100 \mathrm{ng} / \mathrm{mL}$ ), while amylase and carcinoembryonic antigen were low.

Pancreatic endocrine neoplasms are occasionally manifested as cystic lesions [ 1 -4]. Differential diagnosis of pancreatic cystic neoplasms is significantly enhanced by cyst fluid analysis [5].

To our knowledge, this is the first report that demonstrates a high chromogranin A level in the fluid of a cystic pancreatic neuroendocrine tumor sampled during EUS-guided FNA. This can be a useful diagnostic tool confirming a preoperative diagnosis of PEN, especially in those cases

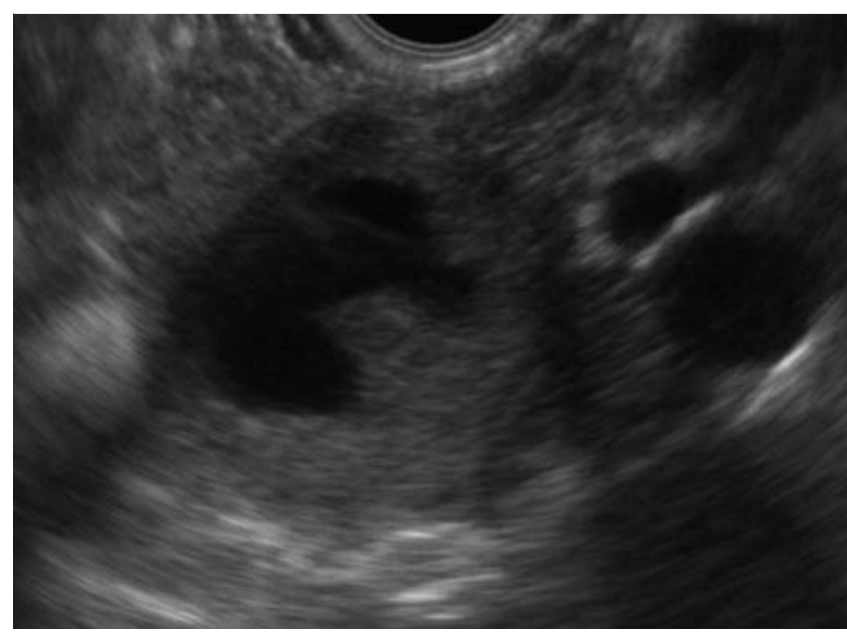

Fig. 1 Endoscopic ultrasound (EUS) image demonstrating the pancreatic lesion, which appeared oval with a thin and hypoechoic rim and a central, anechoic part; the lesion was $22 \times 18 \mathrm{~mm}$.

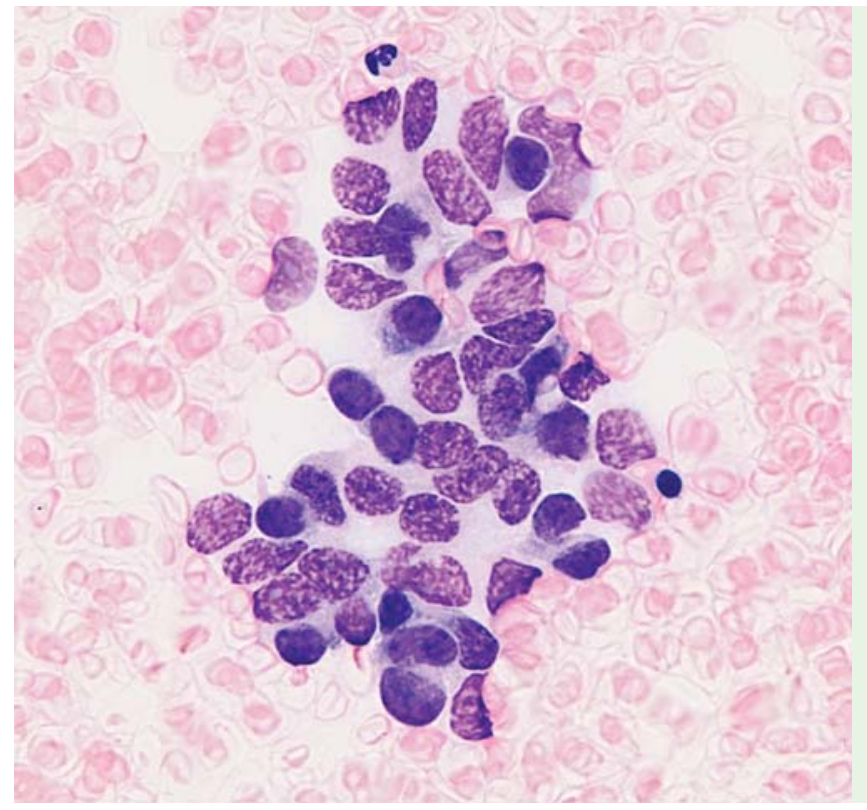

Fig. 2 A direct smear with Giemsa staining showed few epithelial cells with the cytomorphological features of neuroendocrine tumors: smooth, round nuclei, finely stippled chromatin, and delicate cytoplasm. These bland tumor cells seem to be arranged in rosette-like structures.

where FNA gives little material for traditional cytological and ICC investigations.

Endoscopy_UCTN_Code_TTT_1AS_2AD

Endoscopy_UCTN_Code_CCL_1AF_2AZ_3AB

\section{Competing interests: None}

F. Maletta ${ }^{1}$, D. Pacchioni ${ }^{1}$, P. Carucci ${ }^{2}$, G. Accinelli ${ }^{1}$, M. Bruno ${ }^{2}$, F. Brizzi ${ }^{2}$, P. Allegranza², M. Rizzetto ${ }^{2}$, G. Bussolati $^{1}$, C. De Angelis ${ }^{2}$

Department of Biomedical Science and Oncology, University of Turin, Italy
2 Department of Gastrohepatology, University of Turin, Italy

\section{References}

1 Baker MS, Knuth JL, DeWitt J et al. Pancreatic cystic neuroendocrine tumors: preoperative diagnosis with endoscopic ultrasound and fine-needle immunocytology. J Gastrointest Surg 2008; 12: 450-456

2 Bordeianou L, Vagefi PA, Sahani D et al. Cystic pancreatic endocrine neoplasms: a distinct tumor type? J Am Coll Surg 2008; 206: $1154-1158$

3 Goh BK, Ooi LL, Tan YM et al. Clinico-pathological features of cystic pancreatic endocrine neoplasms and a comparison with 


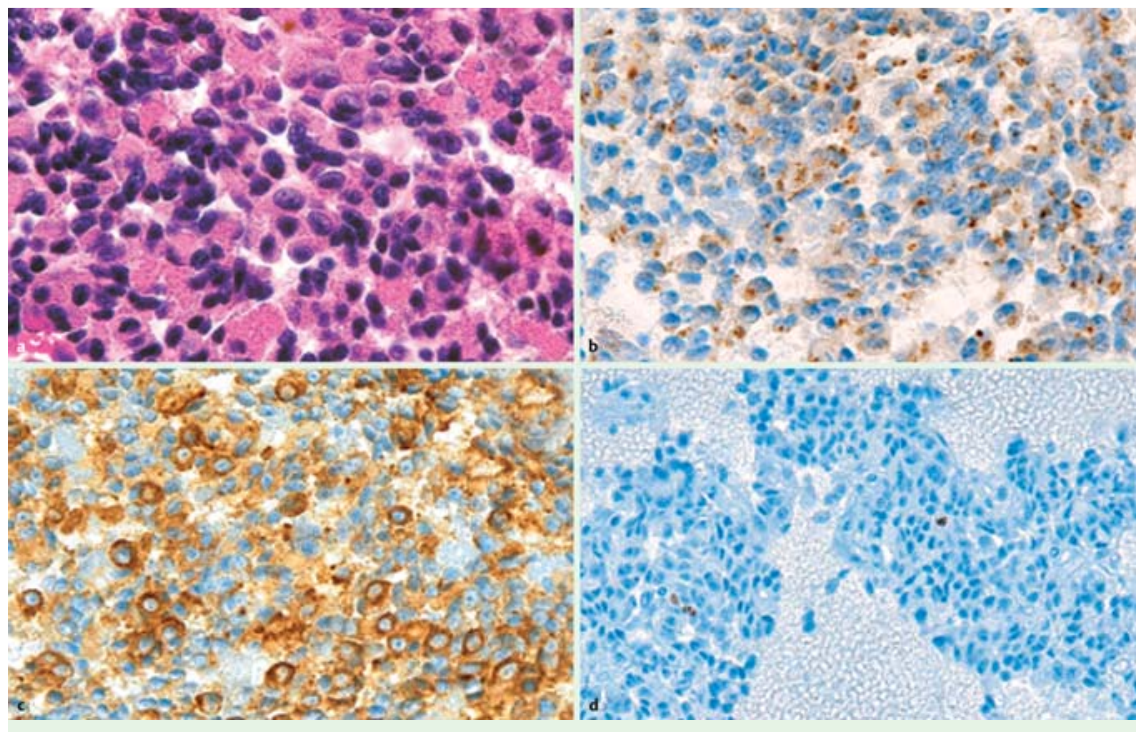

Fig. 3 Cell block preparation showing numerous cells with a plasmacytoid appearance, regular nuclear membrane, and finely granular chromatin pattern. a Hematoxylin and eosin stain. The neuroendocrine differentiation was confirmed by strongly positive immunostaining for $\mathbf{b}$ chromogranin $\mathrm{A}$ and c synaptophysin. $\mathbf{d}$ The proliferative index (Ki-67) was low (<1\%). their solid counterparts. Eur J Surg Oncol 2006; 32: $553-556$

4 Charfi S, Marcy M, Bories E et al. Cystic pancreatic endocrine tumors: an endoscopic ultrasound-guided fine-needle aspiration biopsy study with histologic correlation. Cancer Cytopathol 2009; 117: 203 - 210

5 Linder JD, Geenen JE, Catalano MF. Cyst fluid analysis obtained by EUS-guided FNA in the evaluation of discrete cystic neoplasms of the pancreas: a prospective single-center experience. Gastrointest Endosc 2006; 64: $697-702$

\section{Bibliography}

DOI $10.1055 / \mathrm{s}-0030-1256036$

Endoscopy 2011; 43: E34-E35

(c) Georg Thieme Verlag KG Stuttgart - New York . ISSN 0013-726X

\section{Corresponding author}

C. De Angelis, MD

S.C. Gastro-Epatologia D.U.

Ospedale Molinette

Corso Bramante 88

10126 Torino

Italy

Fax: +39-011-6335927

eusdeang@hotmail.com 